\title{
Comparing Tangible and Fully Virtual Haptic Systems for HMI Studies in Simulated Driving Situations
}

\author{
Antoine Lassagne ${ }^{1,2}$, Andras Kemeny ${ }^{1,2}$, Javier Posselt ${ }^{1}$ and Frederic Merienne ${ }^{2}$ \\ 1. Renault, Autonomous Driving and Virtual Reality, TCR AVA 013, 1 avenue du golf, 78288 Guyancourt, France \\ 2. Le2i FRE 2005, Arts et Metiers, CNRS, Univ. Bourgogne Franche-Comte, HeSam, France
}

\begin{abstract}
The contribution of tangible and intangible feedback is compared for virtual tactile car Human-Machine Interfaces (HMI) design, to measure their performance both in static conditions and while driving. A subjectively calibrated transparent glass provides tangible passive haptics, and visual cues are used to study sensory substitution based intangible interactions. The results show that the performance of the subjects was significantly improved in driving conditions as they interacted faster, more accurately and with a higher satisfaction. In addition, our findings highlight that the contribution of tangible systems is significantly lower in driving conditions, raising new questions about the nature of haptic modalities in the function of the context of use. This study provides additional knowledge about the influence of dynamic environments and external tasks on haptic perception.
\end{abstract}

Key words: Automotive design, HMI, haptics, perception, virtual reality.

\section{Introduction}

Virtual Reality (VR) has many applications in automotive industry, especially in engineering design. However, haptic feedback may offer new capabilities to simulators, allowing effective interactions and improved perception to enhance the immersion [1]. HMI testing is one of many use-cases that inherently requires interactions.

\subsection{Passive Haptics}

Passive haptic consists of opposing tangible objects, co-located with virtual objects which need haptics. Hoffman et al. [2] reached a satisfactory haptic feedback by introducing physical plates into a virtual environment (VE). Carlin et al. [3] evaluated the possibility of curing spider phobia through a similar implementation and also provided promising results. Insko et al. [4] significantly improved presence in a visual-cliff environment augmented by means of passive haptics. They all concluded that passive haptic generates appropriate haptic feedback in various situations, and they emphasize the added value of adding haptic capabilities to VE, especially passive haptic which is safe, affordable, simple to implement and requires no continuous computation.

\subsection{Intangible Haptics}

Previous research has proposed intangible haptic systems with sensory substitution, effective in numerous uses. Realistic visual cues like inter-reflections or shadows [5-7] provided significant performance improvement in interactions. Kitagawa et al. [8] measured the accuracy of subjects applying force in the presence of visual and auditory non-realistic cues and showed that continuous visual cues significantly improve performance, while auditory should be discrete for intrusiveness issues. In similar studies, Petzold et al. [9] concluded that despite the advantages of sensory substitution, there exists an additional cognitive burden of translating sensory information other than haptic into force.

\subsection{Spatial Perception in VEs}

Perception of depth and scale is not always accurate in VEs and it can deteriorate the haptic when not taken into consideration. According to different studies 
[10-12], the environment should include a lot of surrounding objects to enhance spatial perception, and there exist important disparities between subjects. Research also demonstrated that high visual quality may improve spatial perception [13]. In driving simulation, motion parallax and egocentric direction are sources of information, as discussed by Kemeny et al. [14]. CAVE systems bring additional sources of bias.

- Glaze tracking is often interpolated from head tracking. Morphological disparities like interpupillary distance [15], eye depth and nose type lead to errors in the interpolation. Depth and slant distance perception suffer from these disparities.

- Vergence-accommodation conflicts weaken the robustness of spatial perception [16].

Haptic devices require accurate spatial perception to render haptic in coordination with vision. On the other hand, haptics should improve spatial perception by providing more perceptual information to subjects [17, 18].

\subsection{Tactile dashboard HMI design}

Previous results compared tangible and intangible interfaces in static conditions [19]. It provided an evaluation of different haptic systems for tactile dashboard HMI design and highlighted several issues.

- The tangible interface significantly enhanced usability in comparison with the visual-only interface which was difficult to interact with.

- A lack of robustness in human spatial perception was identified as a major obstacle to VEs interaction, especially when no tangible haptic system can provide additional space references.

Subjects were not driving during this experiment. Further studies are thus needed in a driving context to complete this evaluation. The final goal would be to implement such a system as an effective engineering tool.

\subsection{Research Questions}

This study aims at comparing the performances of haptic systems in different contexts in immersive VEs.
We seek to study how a dynamic environment and a driving task can lead to performance differences when compared to the static situation previously studied.

Our questions of research are the following:

- To which extent a dynamic environment and a driving task modify the performance of interactive systems in immersive environments?

- Is this influence different between several haptic modalities with known performances?

We expect major differences in comparison with a static context due to two factors.

- The cognitive burden induced by driving should force subjects to focus and may improve their performance.

- We expect a continuously renewed environment to decrease the perceptual shifts observed in static situations, as demonstrated by Atkins et al. [20, 21].

\section{Methodology}

Eleven subjects (males and females, Renault employees) were instructed to interact with a simple virtual tactile dashboard HMI in the CAVE while driving. The interface displayed 4 buttons with 3 scales. Subjects successively employed intangible and tangible interaction systems to proceed with 2 (intangible, tangible) $* 3$ (button scales) series, in a random order. During series, 20 interactions were called by the HMI (one button turns green until the subject touches it). The flow of series is described in Fig. 1.

As performance indicators, we measured execution duration, relative error, and two questionnaires were filled out by subjects, corresponding to tangible and intangible haptic modes.

These experiments took place in Renault P3I (Industrial Immersive Integration Platform) CAVE, a 4-sided virtual reality room powered by ultra-short throw full HD Panasonic projectors. We use active stereoscopy and optical tracking with A.R.T. infrared technologies. ${ }^{1}$ Interaction implementation is described below:

\footnotetext{
1 http://www.ar-tracking.com/home/.
} 


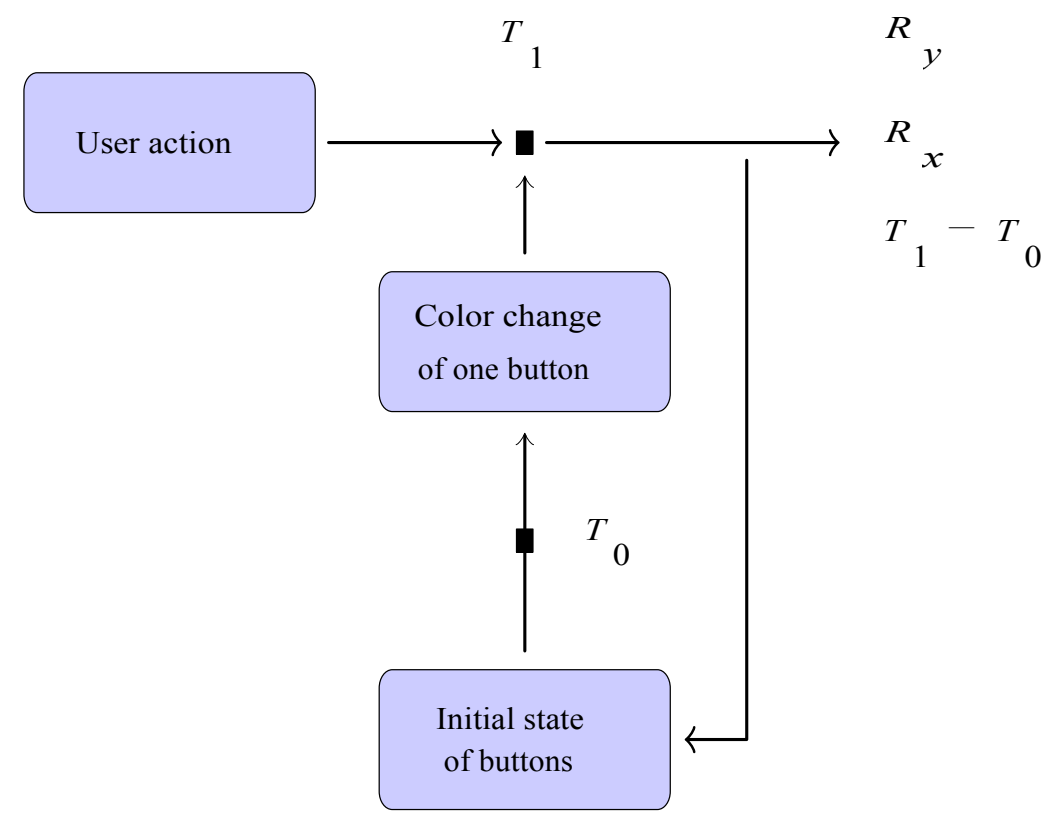

Fig. 1 Flow of a single serie. Relative errors $\left(R_{x}, R_{y}\right)$ represent the relative offset between the location of the interaction and the actual center of the button. $T_{1}-T_{0}$ is the time spent by the user to perform an interaction.

- Intangible feedbacks: color changes from the interface, no haptic feedback.

- Tangible feedback: a passive transparent glass attached to a support is added.

In addition, a 3-finger tracked glove is used to acquire interactions.

The virtual scene was displayed by Oktal SCANeR Studio [22] and placed users inside a car in driving conditions (Fig. 2). Logitech G25 steering wheel was dedicated to driving. Theoretical latency was under $30 \mathrm{~ms}$ with this setup.

The methodology and apparatus were identical during static studies, except for the smallest scale. 17 subjects took part in static studies, 3 of them took part in both experiments.

Subjective calibration Consistency between vision and interactions was attained by means of a subjective calibration. This calibration allowed us to bypass morphologic disparities. Subjects were instructed to successively touch 3 corners of the virtual touchscreen to map the collision engine to their perceived visual location (Fig. 3).

When the transparent glass was in use, it was placed by subjects on what they reported was the right location.
We put a particular attention on this step, as misplaced glasses had highly degraded the experiment during preparation.

\section{Results}

Student's t-Test was used for the statistical analysis of paired samples among the different conditions (tangible or intangible interactions, static or dynamic scenarios), with a significance level of $5 \%$.

\subsection{Execution Duration}

The data comparison of execution duration is displayed in Fig. 4. The static study resulted in significant performance differences between intangible and tangible systems. Driving simulation conditions provided the following results:

- Interactions with the intangible system were significantly ( $\sim 33 \%)$ faster while driving.

- The tangible system had a lower potential for improvement as it already provided low execution duration. It still improved moderately.

- Standard deviation of execution duration without tangible interface has been highly reduced.

Driving conditions led to a higher performance for 


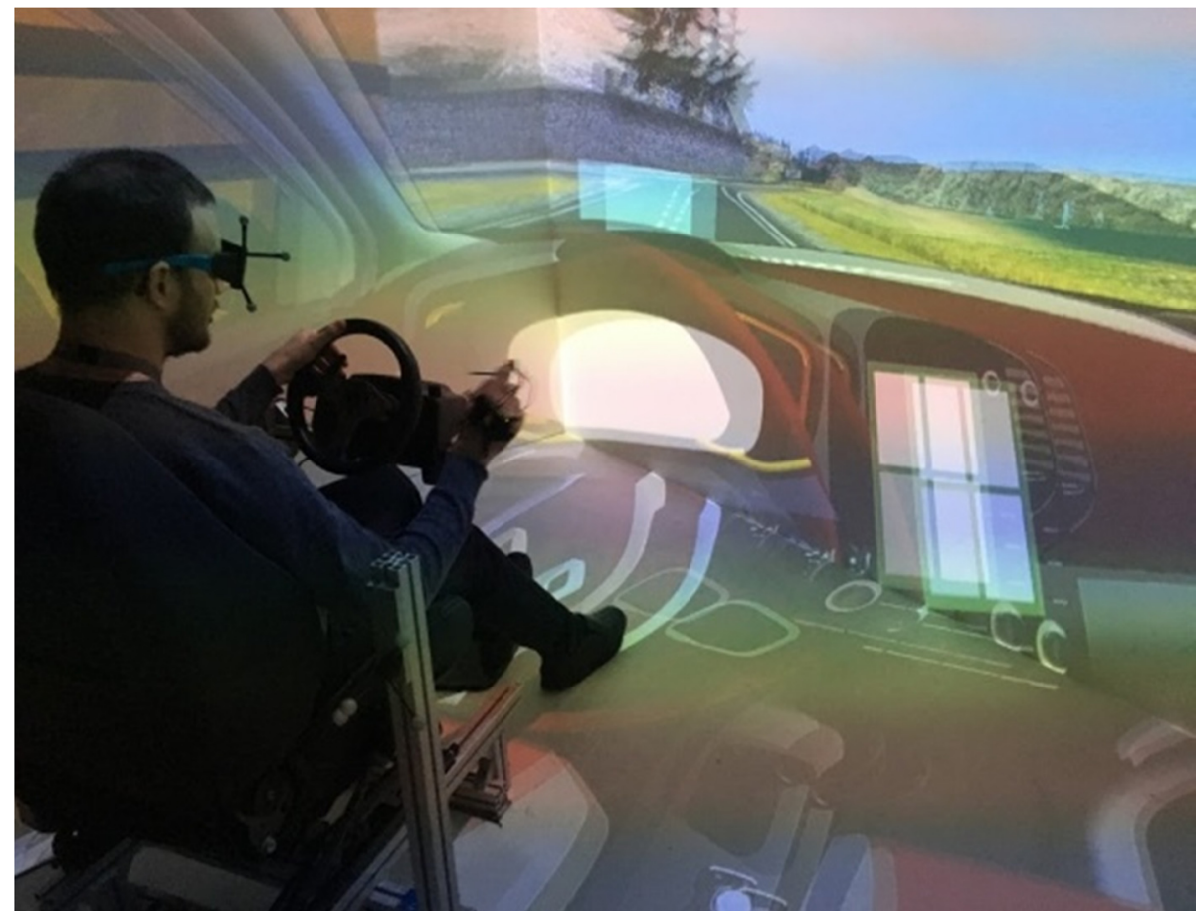

Fig. 2 Subject immersed in the VE.

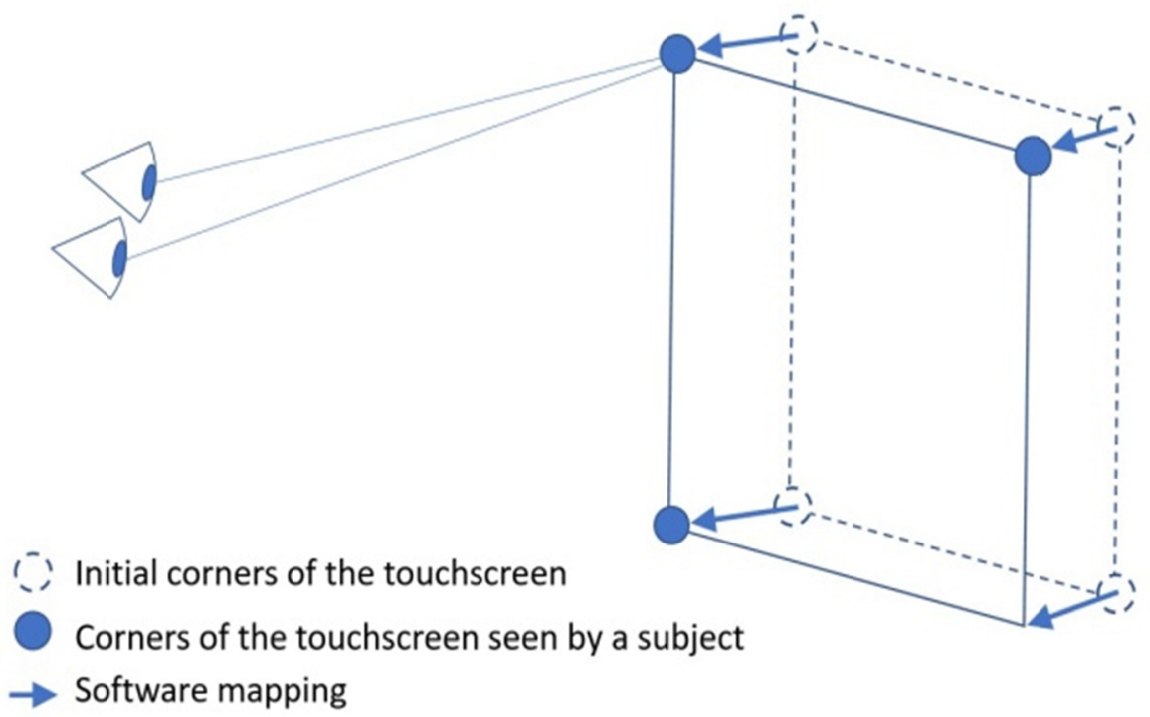

Fig. 3 Subjective calibration allowing accurate visuo-haptic consistency.

both intangible and tangible studies. These conditions were more advantageous to the intangible interaction system which could almost attain the same level of performance as the tangible one.

Robustness issues - low robustness was a major obstacle to subject interaction in static studies. Their execution duration was higher due to spatial perception issues, especially with small buttons that some subjects were unable to interact with at all. In this study, these issues were not encountered. Furthermore, we added a third, smaller button scale which did not cause difficulties to any subject.

Reduced standard deviation also suggests that the system was easier to apprehend as there were less 


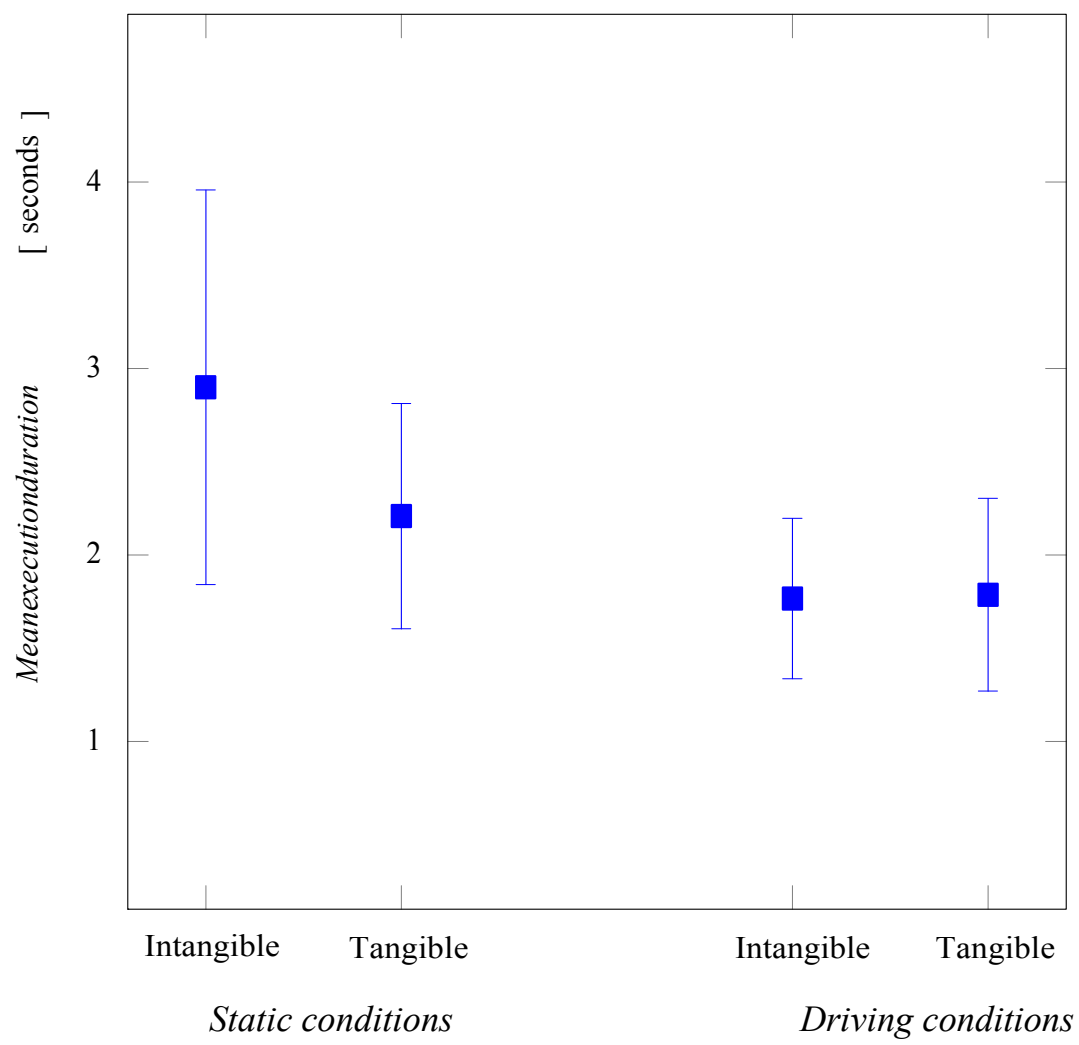

Fig. 4 Mean execution duration difference between static and driving context for tangible and intangible systems.

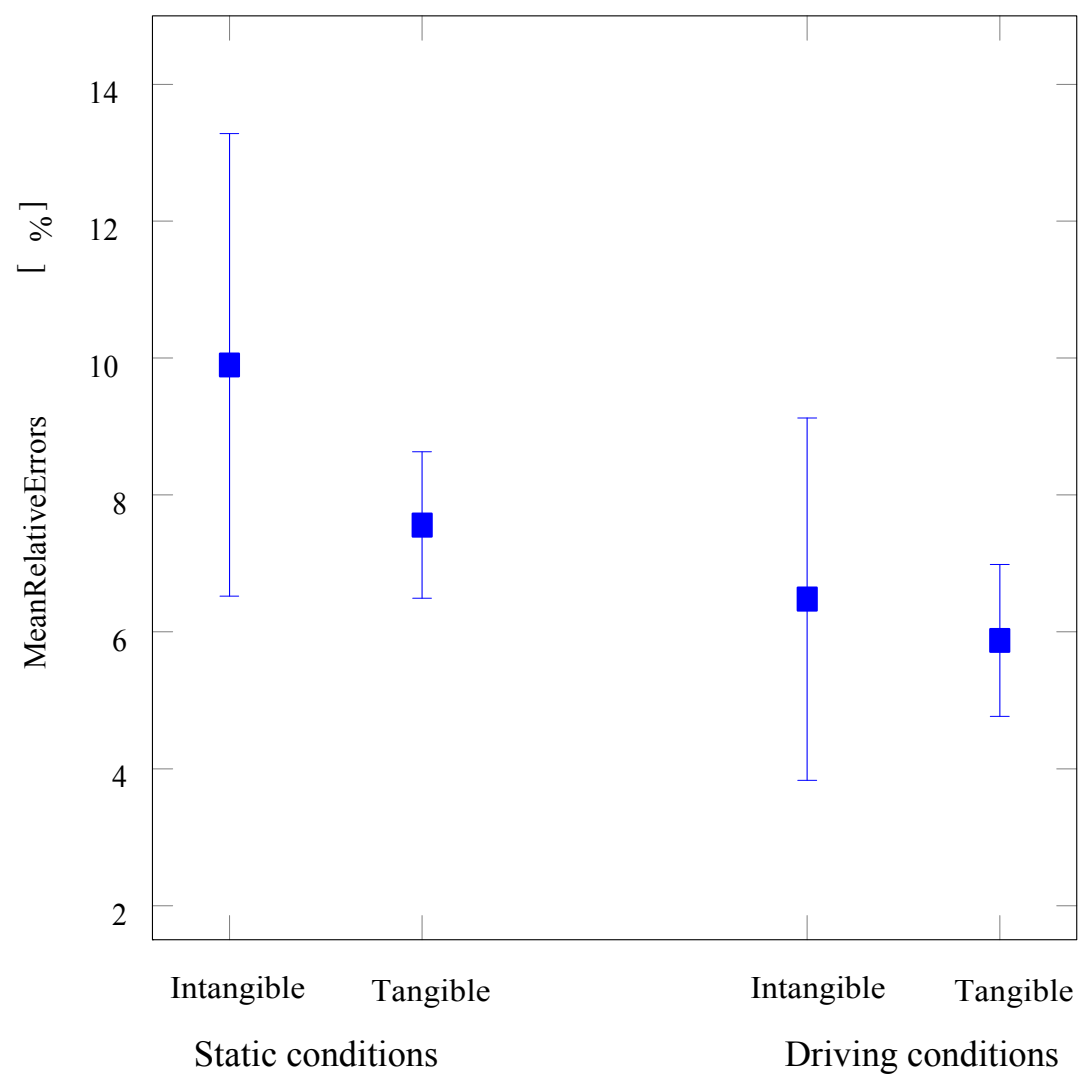

Fig. 5 Mean relative error difference between static and driving context for tangible and intangible systems. 


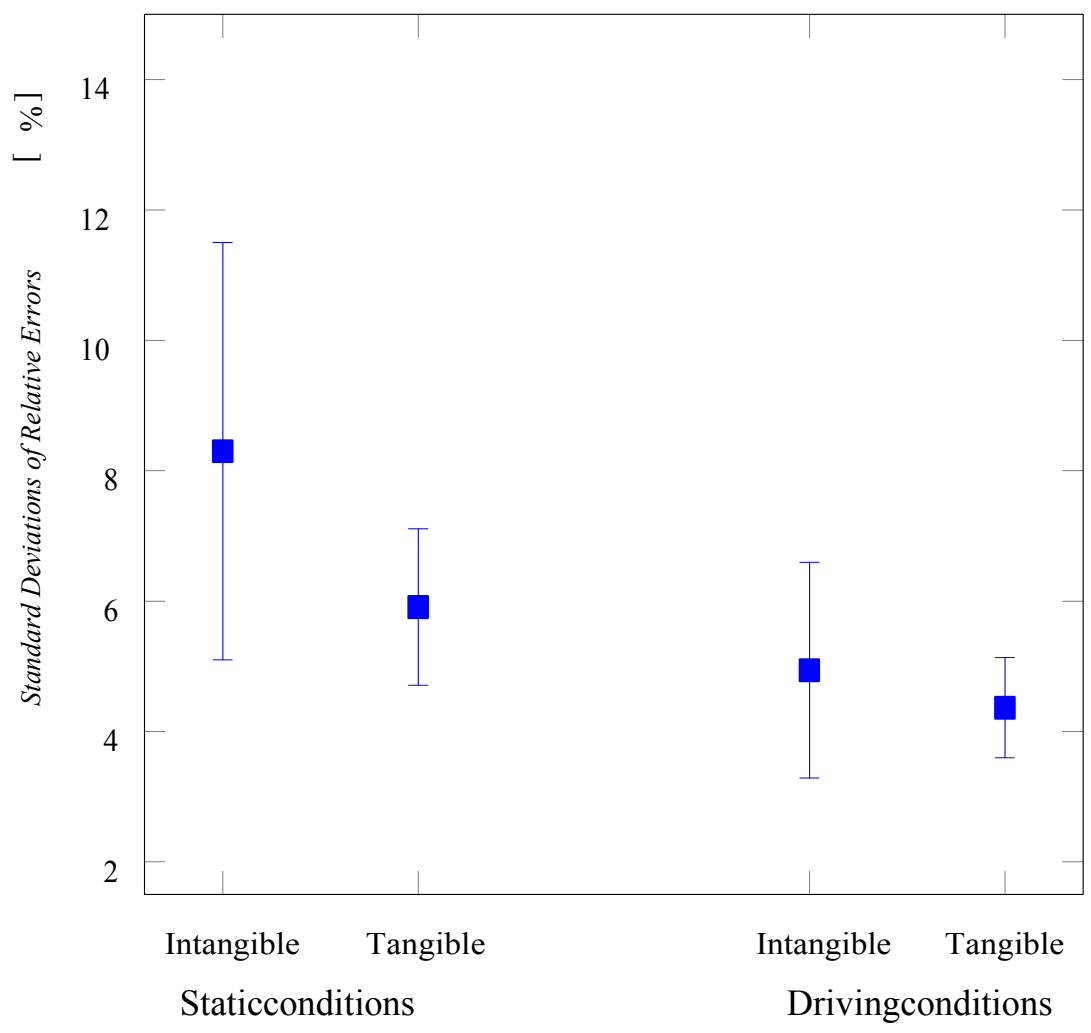

Fig. 6 Mean standard deviations of relative errors.

disparities between subjects.

\subsection{Relative Error}

The data comparison of relative errors is displayed in Fig. 5.

Accuracy - Once more, the difficulties observed with the intangible interface in static mode seem to disappear in driving conditions. Whereas intangible haptics gave a poor performance in static mode, it nearly attains the same level of accuracy as tangible haptics in dynamic mode. Standard deviation is still significantly lower with the tangible system, which also benefited from driving conditions.

Subjects precision and disparities - Standard deviation of relative errors is illustrated in Fig. 6. Disparities were especially reduced in intangible mode, which provided the highest values. Tangible interactions had already provided low disparities in the static context and thus had less potential for progress, nevertheless performance was also improved.

\subsection{Subject Judgement}

All subjects reported that they encountered no difficulties to interact while driving. Their interactions were successful with the smallest button scale, and they were surprised when told that subjects had difficulties with medium scales during static studies. A majority of the subjects reported that they preferred the intangible modality for various reasons:

Easier setup: placing the glass on the right place could be difficult and had an important influence on the experience; therefore 2 to 5 minutes were needed for each subject.

False-positives: when an interaction was failed with the tangible system, subjects felt a haptic feedback anyway. No wrong feedback was produced when the intangible system was used.

Constraints: subjects were foreseeing future use cases involving interactions with other non-flat pieces, which could not always be achieved with a transparent glass. 


\section{Discussion}

\subsection{Spatial Perception}

The main identified influence on performance is the quality of spatial perception, weakened by unreliable perceptual inputs. Inaccurate spatial perception brings inaccurate actions and a reduced confidence, which users encountered in a static context. Several parameters of this experiment led to an improved spatial perception.

Level of concentration on cues affects perception The driving task forced subjects to continuously concentrate on their environment to prevent a crash, whereas they could keep their eyes on the HMI during static studies. Spatial cues are distributed inside the whole VE (objects of known size, parallax, stereoscopy, etc.) and are thus more easily perceived by subjects when they focus on them. Furthermore, these cues are continuously renewed as the car progresses, and they are seen from different points of view, in different layouts. Moving the head also adds parallax cues.

Shifts in visuo-haptic consistency - Morphologic disparities are often responsible for miscalculated vision frustrums. Most of the time, the software uses mean anthropometric data to interpolate the location of the eyes from the location of the tracked 3D glasses, causing inaccurate eye location data. These disparities are valued in millimeters but can become centimeters on the screen.

The subjective calibration used in this experiment grants visuo-haptic consistency in the configuration of the calibration, when looking straight forward. However, while some subjects looked straight forward to interact, others kept their head toward the road and preferred moving only their eyes. Subjective methods are thus not satisfactory, although analytic methods are not without problems. In early experiments, we measured IPD without satisfactory visuohaptic consistency. Further studies will try to improve this aspect by means of continuous recalibration.

\subsection{Choosing the Best Tool for HMI Design in VR}

The added value of tangible interfaces was not probative in the driving simulation context, whereas it significantly improved interaction performance in the static context. The subjects were executing the exact same task and the only difference was the driving conditions. Therefore, the importance of the relationship between haptic modalities and usecases/context must be emphasized. There exists no perfect haptic device for every use, but their individual advantages can be optimized for specific use-cases.

Active or passive scenario - Active and passive actions are handled differently by the brain, as studied by Mima et al. [23]. Different areas and magnitudes are involved, inducing performance disparities, when subjects are acting or not.

- Haptic systems with poor performance should only be used in active scenarios to obtain satisfactory results.

- Haptic systems with assumed higher performance can be used in either active or passive scenarios.

Industrial specificities - The subjects' preferences may have been influenced by their affiliation to the automotive industry. Industrial projects induce additional constraints like non-intrusiveness, high robustness, and a focus on workability rather than realism. Since an interactive device is supposed to be used in a specific context when implemented in an industry, this context should be known with as much precision as possible before choosing a haptic device. Indeed, even a small parameter, like static or driving conditions, can have a significant influence.

\section{Conclusions and Implications}

The comparison of tangible and fully virtual haptic systems for HMI studies brings new data on the contribution of haptic modalities in driving conditions. The respective performances of two haptic implementations, a sensory substitution-based intangible system and a tangible passive haptic system, 
were compared in driving simulations and static contexts by means of efficiency, accuracy and user satisfaction. Results show that subjects significantly improved their performance in driving conditions. Furthermore, the added value of tangible haptics was significantly lower in driving conditions. Our results bring evidence that the evaluation of haptic modalities has to be carried out in driving conditions, as their contribution may be very different in static conditions.

\section{References}

[1] Brooks, F. P. 1999. "What's Real about Virtual Reality?." IEEE Computer Graphics and Applications 19 (6): 16-27.

[2] Hoffman, H. 1998. "Physically Touching Virtual Objects Using Tactile Augmentation Enhances the Realism of Virtual Environments.” IEEE Comput. Soc, 59-63.

[3] Carlin, S., Hoffman, H. G., and Weghorst, S. 1997. "Virtual Reality and Tactile Augmentation in the Treatment of Spider Phobia: A Case Report." Behaviour Research and Therapy 35 (2): 153-8.

[4] Insko, B. E. 2001. "Passive Haptics Significantly Enhances Virtual Environments.” Ph.D. thesis, University of North Carolina at Chapel Hill.

[5] Chan, L.-W., Kao, H.-S., Chen, M. Y., Lee, M.-S., Hsu, J., and Hung, Y.-P. 2010. Touching the Void: Direct-Touch Interaction for Intangible Displays. 2625, ACM Press.

[6] Hu, H. H., Gooch, A. A., Thompson, W. B., Smits, B. E., Rieser, J. J., and Shirley, P. 2000. "Visual Cues for Imminent Object Contact in Realistic Virtual Environment." In Proceedings of the conference on Visualization'00, 179-85, IEEE Computer Society Press.

[7] Madison, C., Thompson, W., Kersten, D., Shirley, P., and Smits, B. 2001. "Use of Inter-reflection and Shadow for Surface Contact." Perception \& Psychophysics 63 (2): 187-94.

[8] Kitagawa, M., Dokko, D., Okamura, A. M., Bethea, B. T., and Yuh, D. D. 2004. "Effect of Sensory Substitution on Suture Manipulation Forces for Surgical Teleoperation." Studies in Health Technology and Informatics, 157-63.

[9] Petzold, B., Zaeh, M. F., Faerber, B., Deml, B., Egermeier, H., Schilp, J., and Clarke, S. 2004. "A Study on Visual, Auditory, and Haptic Feedback for Assembly Tasks." Presence: Teleoperators and Virtual Environments 13 (1): 16-21.
[10] Cutting, J. E., and Vishton, P. M. 1995. "Perceiving Layout and Knowing Distances." In Perception of Space and Motion, 69-117. Elsevier. ISBN 978-0-12-240530-3.

[11] Kenyon, R. V., Sandin, D., Smith, R. C., Pawlicki, R., and Defanti, T. 2007. "Size-Constancy in the CAVE." Presence: Teleoperators and Virtual Environments 16 (2): 172-87.

[12] Loomis, J. M., Silva, J. A. D., Philbeck, J. W., and Fukusima, S. S. 1996. "Visual Perception of Location and Distance." Current Directions in Psychological Science 5 (3): 72-7.

[13] Interrante, V., Ries, B., and Anderson, L. 2006. Distance Perception in Immersive Virtual Environments, 3-10, IEEE, 2006, ISBN 978-1-4244-0224-3.

[14] Kemeny, A., and Panerai, F. 2003. "Evaluating Perception in Driving Simulation Experiments." Trends in Cognitive Sciences 7 (1): 31-7.

[15] Dodgson, N. A. 2004. "Variation and Extrema of Human Interpupillary Distance." In Proceedings of SPIE - The International Society for Optical Engineering, 36-46.

[16] Wann, J. P., Rushton, S., and Mon-Williams, M. 1995. "Natural Problems for Stereoscopic Depth Perception in Virtual Environments." Vision Research 35 (19): 2731-6.

[17] Burge, J., Girshick, A. R., and Banks, M. S. 2010. "Visual-Haptic Adaptation Is Determined by Relative Reliability." Journal of Neuroscience 30 (22): 7714-21.

[18] Ernst, M. O., and Bulthoff, H. H. 2004. "Merging the Senses into a Robust Percept." Trends in Cognitive Sciences 8 (4): 162-9.

[19] Lassagne, A., Kemeny, A., Posselt, J., and Merienne, F. 2017. Performance of Different Haptics Systems for Tactile Dashboard HMI Design in CAVEs. (In press)

[20] Atkins, J. E., Fiser, J., and Jacobs, R. A. 2001. "Experience-Dependent Visual Cue Integration Based on Consistencies between Visual and Haptic Percepts." Vision Research 41 (4): 449-61.

[21] Atkins, J. E., Jacobs, R. A., and Knill, D. C. 2003. "Experience-Dependent Visual Cue Recalibration Based on Discrepancies between Visual and Haptic Percepts." Vision Research 43 (25): 2603-13.

[22] Kemeny, A. 1993. "A Cooperative Driving Simulator." In Proceedings of the International Training Equipment Conference (ITEC), 67-71.

[23] Mima, T. 1999. "Brain Structures Related to Active and Passive Finger Movements in Man.” Brain 122 (10): 1989-97. 